\title{
GaMnAs: Layers, Wires and Dots
}

\section{J. SADOWSKI}

Institute of Physics, Polish Academy of Sciences

al. Lotników 32/46, 02-668 Warszawa, Poland

and MAX-Lab, Lund University, 22100 Lund, Sweden

\begin{abstract}
Thin layers of GaMnAs ferromagnetic semiconductor grown by molecular beam epitaxy on $\operatorname{GaAs}(001)$ substrates were studied. To improve their magnetic properties the post-growth annealing procedures were applied, using the surface passivation layers of amorphous arsenic. This post-growth treatment effectively increases the ferromagnetic-to-paramagnetic phase transition temperature in GaMnAs, and provides surface-rich MnAs layer which can be used for formation of low-dimensional structures such as superlattices. If the surface rich MnAs layer consists of MnAs dots, then it is possible to grow Mn-doped GaAs nanowires.
\end{abstract}

PACS numbers: 75.50.Pp, 62.23.Hj, 81.16.Hc

\section{Introduction}

After the discovery of ferromagnetic phase transition in GaMnAs ternary semiconductor reported by Ohno et al. in 1996 [1] this compound became the most comprehensively studied ferromagnetic semiconductor with ferromagnetic phase transition induced by interactions between spins of Mn ions and free carriers (valence band holes). Mn in GaAs lattice constitutes a source of uncompensated spins and at the same time is an efficient $p$-type dopand, providing concentration of holes in the range of $10^{20}-10^{21} \mathrm{~cm}^{-3}$ for $\mathrm{Mn}$ content of a few percent. The preparation of GaMnAs requires low temperature (LT) molecular beam epitaxial (MBE) growth technique. Use of very low growth temperatures, much lower than usually applied for GaAs induces formation of compensating defects such as arsenic antisites and Mn interstitials. Both defects are double donors, decreasing the concentration of holes provided by $\mathrm{Mn}_{\mathrm{Ga}}$ acceptors [2,3]. Concentration of the first defect can be minimized by an appropriate choice of the growth parameters [4], the latter can be removed by suitable annealing procedures applied after the MBE growth [5, 6]. With the use of optimized growth and post-growth annealing procedures a ferromagnetic phase transition temperature $\left(T_{\mathrm{c}}\right)$ close to $180 \mathrm{~K}$ has been attained recently in GaMnAs [7]. Further $T_{\mathrm{c}}$ increase will probably demand 
increasing Mn content above 10\%, accompanied by the sufficiently high concentration of holes. Recently it was reported that uniform GaMnAs can be grown with Mn content of up to $20 \%$ [8-10], so progress in this direction cannot be excluded.

Besides GaMnAs in form of layers, also low-dimensional structures such as quantum wires have been studied. So far they were fabricated from layered structures with use of e-beam lithography [11-13]. In addition to structures obtained in this way it is possible to obtain self-assembled one-dimensional structures (nanowires, NWs). They are generated due to the catalyzing properties of MnAs nano-islands formed at the GaMnAs surface during MBE growth above the phase separation threshold [14]. In GaAs:Mn nanowires with sufficiently small diameters GaAs occurs in the hexagonal, wurzite phase [15], so far GaMnAs in this structure has not been studied.

\section{Basic properties of GaMnAs}

\subsection{Epitaxial growth of GaMnAs}

GaMnAs can be grown by MBE on GaAs substrates when the substrate temperature during the growth process is much lower than usually applied for GaAs. In the latter case the MBE growth is usually performed with the substrate temperature in the range $590-640^{\circ} \mathrm{C}$, however for GaMnAs much lower temperatures must be used in order to avoid segregation of MnAs clusters. The segregation of $\mathrm{MnAs}$ is easily detectable in reflection high energy electron diffraction (RHEED) images recorded during the MBE growth. Figure 1 shows an example of RHEED pictures from smooth, uniform GaMnAs film, and from a GaMnAs with surface segregated MnAs clusters.

In order to avoid segregation of MnAs the MBE growth of GaMnAs with Mn content higher than 1 at.\% must be performed below $300^{\circ} \mathrm{C}$. The low growth temperature implies generation of structural defects such as As antisites $\left(\mathrm{As}_{\mathrm{Ga}}\right)$ with maximum concentrations reaching 0.5 at.\% [16]. As $\mathrm{As}_{\mathrm{Ga}}$ is a deep donor in
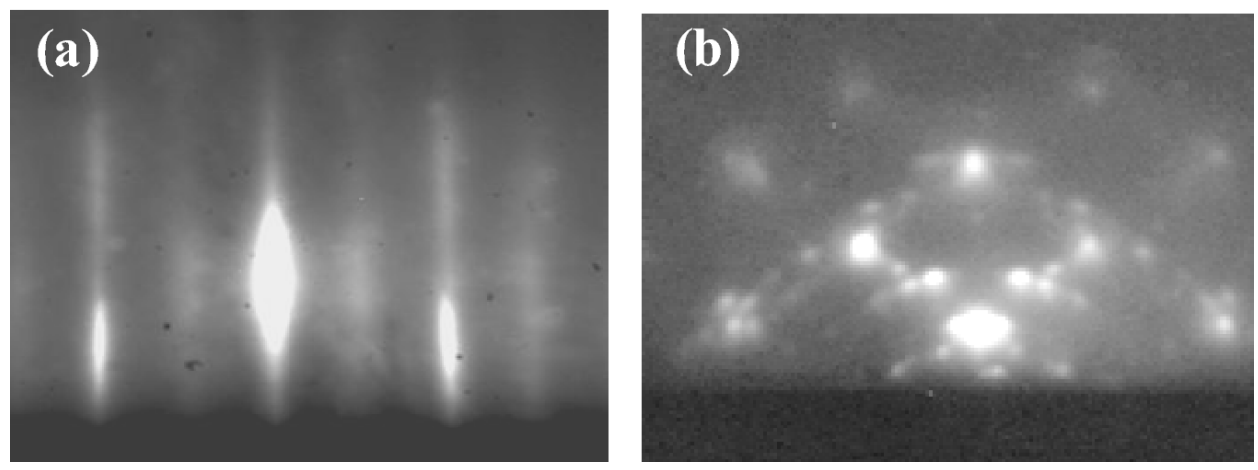

Fig. 1. RHEED diffraction images from the surface of smooth $\mathrm{Ga}_{0.94} \mathrm{Mn}_{0.06}$ As layer (a) and $\mathrm{Ga}_{0.94} \mathrm{Mn}_{0.06} \mathrm{As}$ with surface segregated MnAs islands (b). The direction of e-beam is parallel to the $[-110]$ azimuth of $\operatorname{GaAs}(001)$ substrate. 
GaAs, so it compensates part of the $\mathrm{Mn}_{\mathrm{Ga}}$ acceptors. The presence of $\mathrm{As}_{\mathrm{Ga}}$ cannot be avoided under LT growth conditions. Concentration of this defect can be minimized by using suitable MBE growth conditions, i.e. maximum substrate temperature, slightly below the MnAs segregation threshold [17], and close to stoichiometric supply of the two elements, i.e. low As/Ga flux ratio. A schematic diagram describing GaMnAs growth is shown in Fig. 2.

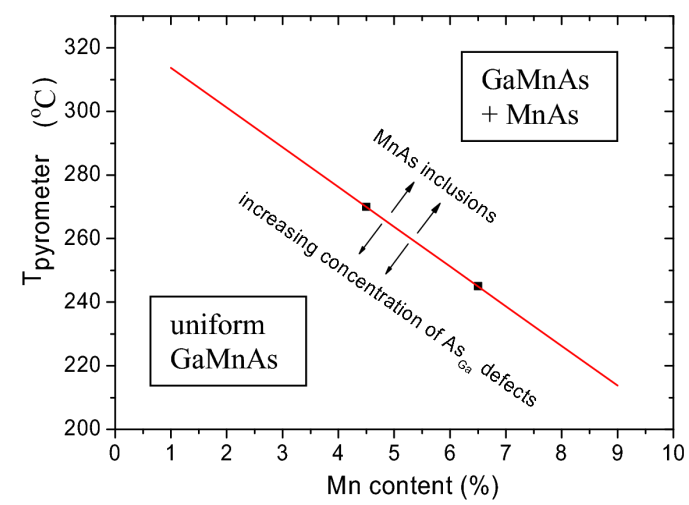

Fig. 2. Simplified diagram for GaMnAs growth. The optimum growth conditions are close to the line separating regions of single phase and MnAs segregation growth.

Another defect, specific for GaMnAs, is Mn located at interstitial sites $\left(\mathrm{Mn}_{\mathrm{I}}\right)$. It has been reported that up to $20 \%$ of a total Mn content in GaMnAs can be located in interstitial sites [18]. Since $\mathrm{Mn}_{\mathrm{I}}$ also behaves as a double donor, it considerably lowers the concentration of holes in GaMnAs, decreasing the temperature of paramagnetic-to-ferromagnetic phase transition.

\subsection{Post-growth annealing}

In contrast to $\mathrm{As}_{\mathrm{Ga}}$ the $\mathrm{Mn}_{\mathrm{I}}$ defects can be removed from the GaMnAs volume by applying post-growth annealing. Due to the post-growth annealing the weakly bounded Mn interstitials diffuse to the GaMnAs surface and are passivated either by oxidation, or by binding with another reactive element [19], such as As deposited on GaMnAs surface as thick amorphous layer directly after the MBE growth. The first method requires very long annealing times (up to $100 \mathrm{~h}$ ) and gives considerable increase in $T_{\mathrm{c}}$ from 60 to $100 \mathrm{~K}$ for as grown GaMnAs up to 170-180 K after annealing. The post-growth annealing method using amorphous As passivation layer developed by the author and co-workers allows to use much shorter annealing times, usually 1-3 h, and for sufficiently thin layers the surface of the annealed GaMnAs is well ordered and suitable for further epitaxial growth. The idea of this annealing method is schematically presented in Fig. 3.

The efficiency of the annealing process is confirmed by magnetization measurements of GaMnAs prior to annealing and after annealing shown in Fig. 4. The 


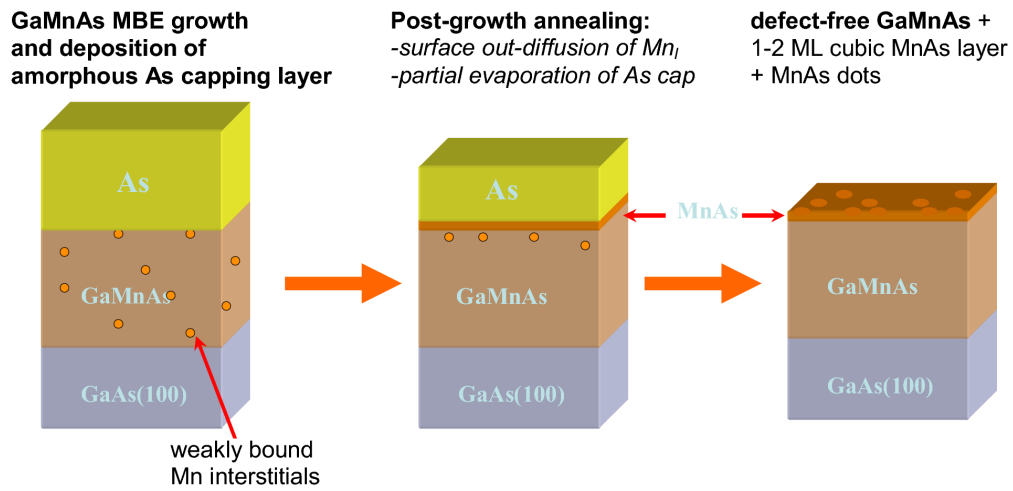

Fig. 3. Procedure of removing Mn interstitials from GaMnAs by post-growth annealing with passivating amorphous arsenic layer.
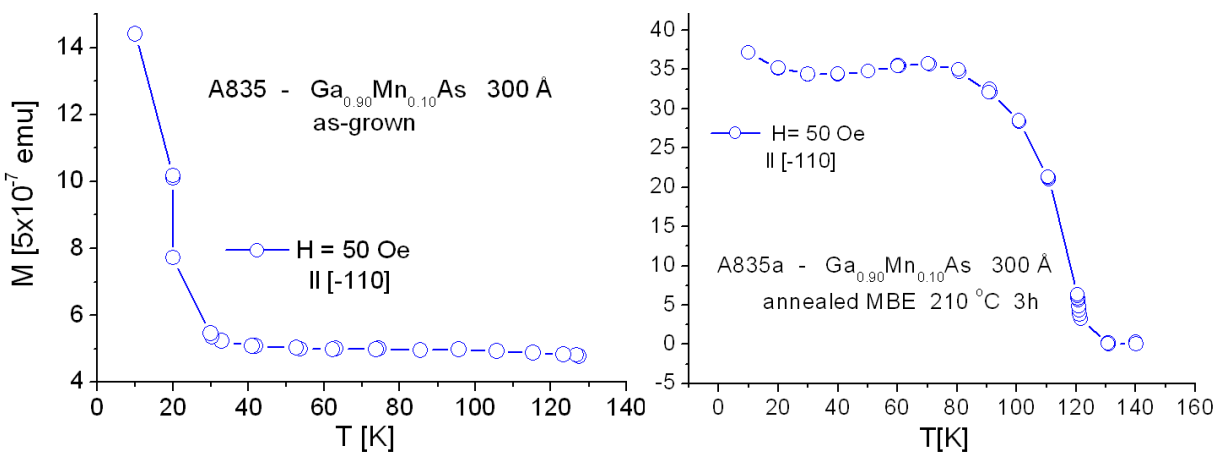

Fig. 4. Temperature dependence of magnetization measured by SQUID for as-grown $300 \AA$ thick $\mathrm{Ga}_{0.9} \mathrm{Mn}_{0.1}$ As layer before annealing (left part) and after annealing with passivating As layer (right part).

remarkable increase in $T_{\mathrm{c}}$ from about 30 to about $130 \mathrm{~K}$ after $3 \mathrm{~h}$ of annealing proves high efficiency of the annealing process.

Since $\mathrm{Mn}$ interstitials are double donors partially compensating $\mathrm{Mn}_{\mathrm{Ga}}$ acceptors, annealing increases the concentration of holes 2-3 times [20]. The saturation moment also increases significantly, since Mn interstitials tend to couple antiferromagnetically with $\mathrm{Mn}$ at $\mathrm{Ga}$ sites making part of $\mathrm{Mn}_{\mathrm{Ga}}$ atoms magnetically passive.

Besides magnetic properties, post-growth annealing also affects the structural parameters of GaMnAs. Mn causes a slight increase of the GaAs lattice constant. According to the theoretical model by Masek et al. [21] the main contribution to the lattice constant increase is due to Mn interstitials. Thus it is expected that upon annealing the GaMnAs lattice constant will be reduced. Figure 5 shows results of X-ray diffraction measurements for a $700 \AA$ thick $\mathrm{Ga}_{0.94} \mathrm{Mn}_{0.06} \mathrm{As}$ layer. The lattice constant reduction can be seen as shifts of diffraction peaks from 


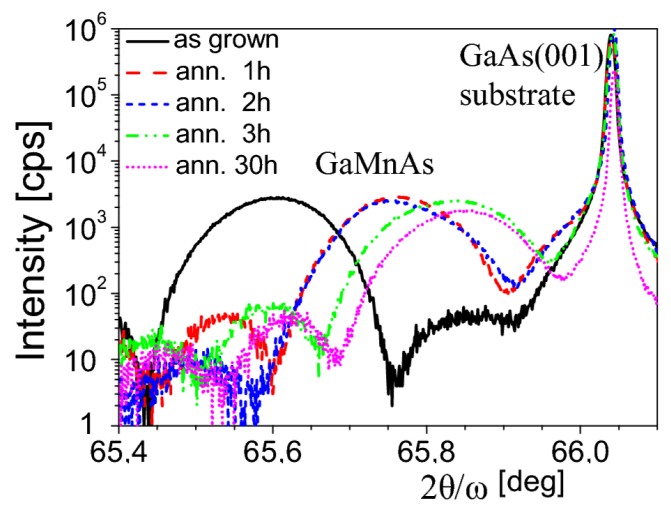

Fig. 5. (004) Bragg reflection from $700 \AA$ thick $\mathrm{Ga}_{0.94} \mathrm{Mn}_{0.06}$ As layer grown on $\operatorname{GaAs}(001)$ substrate before annealing, and after post-growth annealing at $190^{\circ} \mathrm{C}$ for $1,2,3$ and $30 \mathrm{~h}$.

GaMnAs layer to the higher diffraction angles. Already after $1 \mathrm{~h}$ annealing there is a remarkable lattice constant reduction. The effects saturates after annealing for $3 \mathrm{~h}$. Annealing for longer time $(30 \mathrm{~h})$ does not lead to further lattice constant reduction. The broadening of the GaMnAs diffraction peaks is due to the small layer thickness.

It was observed that post-growth annealing of GaMnAs is only efficient for the layers which are not too thick. The thickness limit for the annealing process is close to $1000 \AA$ [6]. It has been tentatively explained by formation of surface layer blocking further out-diffusion of remaining Mn interstitial atoms. In case of annealing in air this is not well understood since the structure of GaMnAs surface oxidized layer is not well known. In case of annealing in high vacuum, with the presence of As annealing leads to formation of a thin MnAs layer [20], in the zinc-blende phase, with the thickness of 1-2 ML. For higher amount of Mn interstitials formation of MnAs quantum dots was detected [22]. Figure 6 shows comparison of surface morphologies of $200 \AA$ thick and $500 \AA$ thick GaMnAs films after annealing with As capping layer. At the end of annealing process the As capping was completely desorbed revealing the GaMnAs surface morphology.

The sequence of GaMnAs growth, As capping and annealing in the MBE system can be repeated leading to the GaMnAs/MnAs(zinc-blende) superlattice structure. Figure 7 shows the results of XRD and magnetization measurements of superlattice structure with $\mathrm{Ga}_{0.93} \mathrm{Mn}_{0.07} \mathrm{As}(75 \AA) / \mathrm{MnAs}$ sequence repeated 8 times. Satellite peaks in X-ray diffraction clearly prove the presence of a superstructure.

The shape of the $M(T)$ curve shown in Fig. 7b is rather untypical, this may be due to the interactions between two magnetic systems i.e. GaMnAs and MnAs layers. 

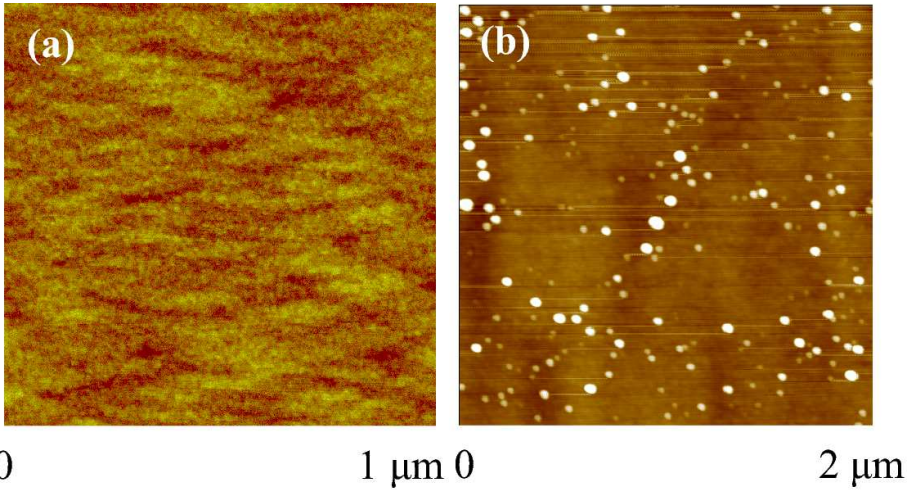

Fig. 6. AFM images of (a) $200 \AA$ thick, (b) $500 \AA$ thick $\mathrm{Ga}_{0.94} \mathrm{Mn}_{0.06}$ As after annealing with As capping. At the end of annealing the As capping layer was completely desorbed.
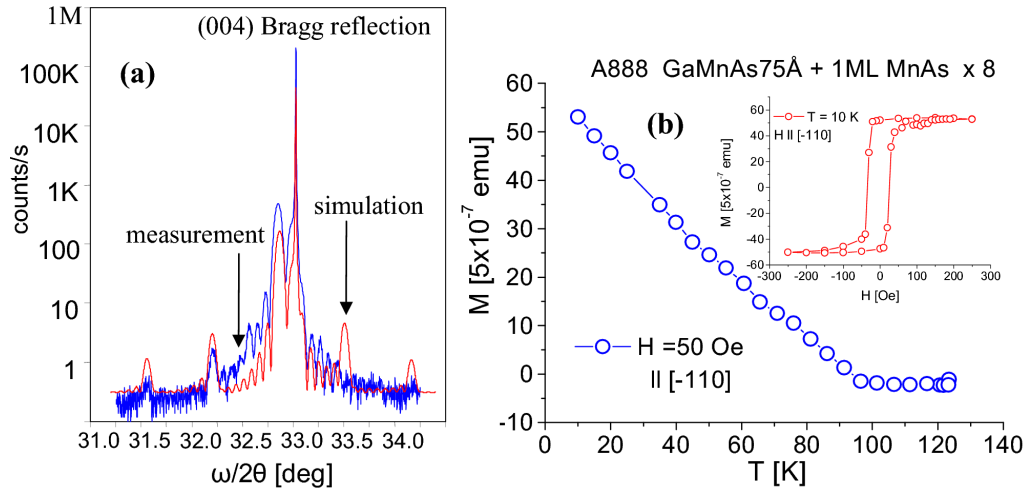

Fig. 7. (a) X-ray diffraction picture from $\mathrm{Ga}_{0.93} \mathrm{Mn}_{0.07} \mathrm{As} / \mathrm{MnAs}(1 \mathrm{ML}) \times 8$ superlattice fabricated by subsequent GaMnAs MBE growth, As capping and annealing; (b) temperature dependence of magnetization and hysteresis loop (inset).

Annealing with surface As passivation also shows a reduced efficiency for layers thicker than about $1000 \AA$, which can be interpreted as blocking further $\mathrm{Mn}_{\mathrm{I}}$ surface out-diffusion by the surface MnAs layer. It is well known that capping GaMnAs even with very thin GaAs layers (5-10 ML) considerably decreases the post-growth annealing efficiency. Recently the nice solution of this problem has been proposed by the Prague-Nottingham group [7]. In this method GaMnAs is annealed, then the surface layer is etched by chemical methods and the next annealing step is performed. Using this method a record $T_{\mathrm{c}}$ of $180 \mathrm{~K}$ was reported.

\section{GaMnAs layers under tensile strain}

GaMnAs grown on $\operatorname{GaAs}(001)$ substrate is in a compressive strain state, which implies that the easy magnetic axis is in-plane (can be also out-of-plane in some narrow range of GaMnAs parameters such as Mn content and concentration 



Fig. 8. (a) $2 \theta / \omega$ scan around (004) Bragg reflection from $500 \AA$ thick $\mathrm{Ga}_{0.925} \mathrm{Mn}_{0.075} \mathrm{As}$ layer deposited on $1.5 \mu$ m thick $\operatorname{In}_{0.07} \mathrm{Ga}_{0.93} \mathrm{As}$ buffer; (b) reciprocal space map of asymmetrical (224) Bragg reflection.
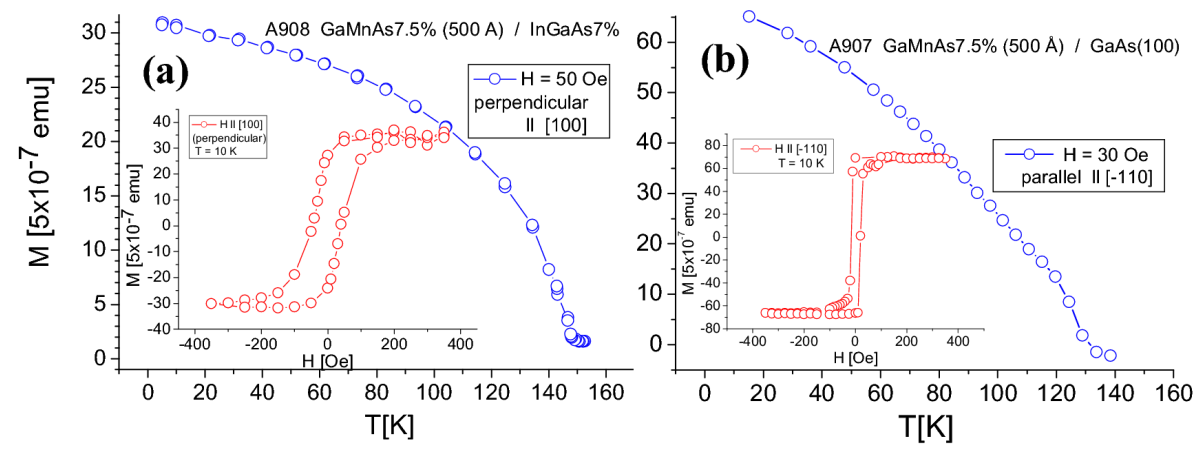

Fig. 9. (a) $M(T)$ curve and hysteresis loop for $\mathrm{Ga}_{0.925} \mathrm{Mn}_{0.075}$ As layer deposited on $1.5 \mu \mathrm{m}$ thick $\operatorname{In}_{0.07} \mathrm{Ga}_{0.93}$ As buffer. The sample has a perpendicular easy magnetization axis; (b) $M(T)$ curve and hysteresis loop for identical sample grown without InGaAs buffer, having an in-plane easy magnetization axis. Samples were annealed simultaneously with As passivation layer directly after the MBE growth.

of holes). In many cases it is desirable to use layers with perpendicular easy magnetization axis. This can be easily achieved in GaMnAs under tensile strain state, i.e. for growth on a surface with larger lattice constant. Using GaAs as initial substrate, a buffer layer with larger lattice constant is obtained by deposition of InGaAs. Figure 8 shows the results of XRD measurements for $500 \AA$ thick $\mathrm{Ga}_{0.925} \mathrm{Mn}_{0.075}$ As layer deposited on a $1.5 \mu \mathrm{m}$ thick $\mathrm{In}_{0.07} \mathrm{Ga}_{0.93}$ As buffer layer. In such a case typical $2 \theta / \omega$ diffraction scan around (004) Bragg reflection is not sufficient, since the perpendicular lattice parameter of GaMnAs is now smaller than the in-plane lattice parameter. In a particular case it can be very close to the lattice parameter of GaAs(001) substrate which gives much higher diffraction intensity. Much more detailed information concerning strain state as well as in- 


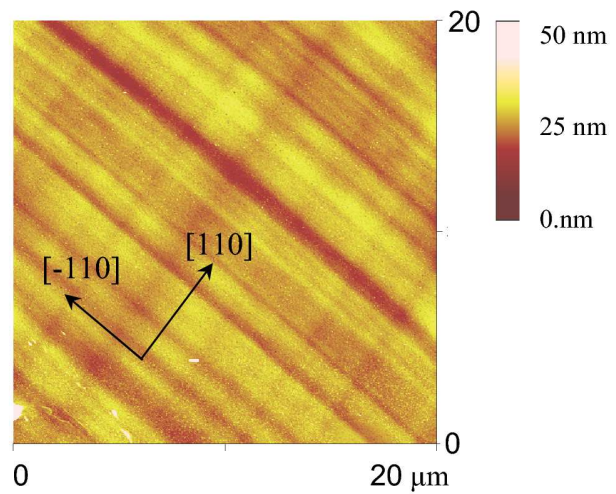

Fig. 10. AFM image of $500 \AA$ thick $\mathrm{Ga}_{0.94} \mathrm{Mn}_{0.06}$ As layer deposited on $\operatorname{In}_{0.11} \mathrm{Ga}_{0.89} \mathrm{As}$ buffer. Cross-hatched surface wedges parallel to [110] and [-110] crystallographic directions are about $50 \AA$ deep.

plane and out-of-plane lattice parameters can be obtained from reciprocal space maps of asymmetrical Bragg reflections. Figure $8 \mathrm{~b}$ shows a reciprocal space map for the same sample as shown in Fig. 8a. 100\% relaxation line (solid line) shows that InGaAs buffer is only partially relaxed. GaMnAs is however completely strained to the InGaAs buffer (i.e. it has the same in-plane lattice constant as InGaAs) as shown by the dashed line.

The sample used for XRD measurements shown in Fig. 8 was subjected to the As capping and post-growth annealing, in the MBE system for $1 \mathrm{~h} 45 \mathrm{~min}$ at $210^{\circ} \mathrm{C}$. The magnetic properties of this sample i.e. temperature dependence of magnetization and hysteresis loop are shown in Fig. 9a. For comparison, Fig. 9b shows results of the same measurements of the twin sample, grown in the same MBE growth run, but without the InGaAs buffer. The higher Curie temperature of sample grown on InGaAs buffer proves the higher efficiency of the annealing process in this case.

The higher annealing efficiency of GaMnAs grown on InGaAs buffer may be due to the surface structure, which in case of InGaAs buffer layers exhibits a micrometer scale corrugation in form of cross-hatched wedges parallel to $[-110]$ and [110] crystallographic directions.

Figure 10 shows an AFM image of a $500 \AA$ thick $\mathrm{Ga}_{0.94} \mathrm{Mn}_{0.06}$ As layer deposited on $\operatorname{In}_{0.11} \mathrm{Ga}_{0.89}$ As buffer. The surface structure of thick InGaAs buffer is also present at the surface of GaMnAs layer.

\section{Surface MnAs clusters as nanowire growth catalyst}

As observed by the author [14] continuing GaMnAs MBE growth in the presence of surface MnAs clusters, i.e. at phase segregation conditions, leads to formation of nanowires. If the MBE growth is performed at too high substrate temperature or too high Mn flux the MnAs clusters are formed at the surface of GaMnAs layer. This can be seen in Fig. 11 showing AFM image of such a surface. 


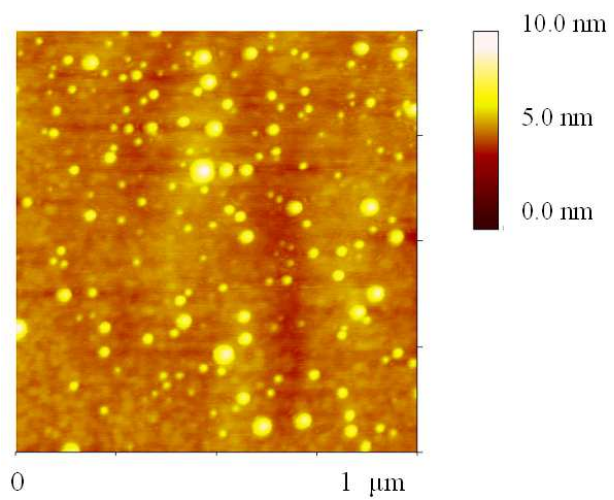

Fig. 11. AFM image of GaMnAs surface with segregated MnAs dots.
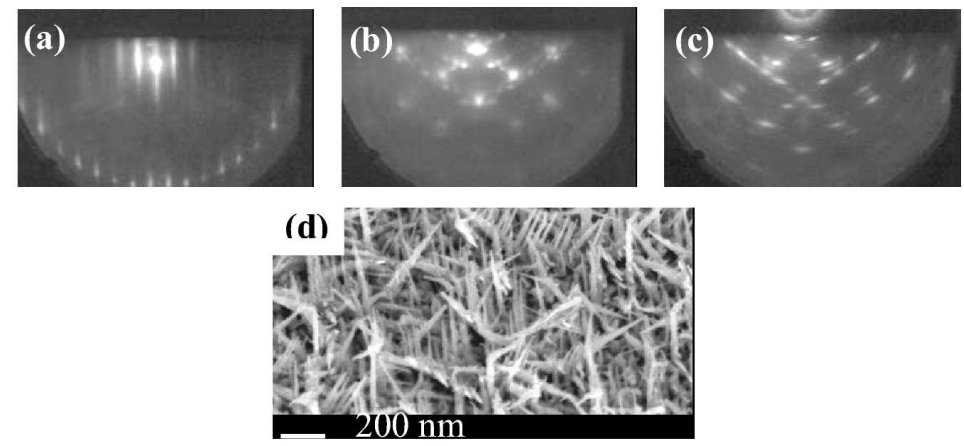

Fig. 12. (a)-(c) RHEED diffraction images recorded during 3D growth mode of GaMnAs nanowires. (a) Initial GaAs(100) surface in [110] azimuth, (b) and (c) diffraction images after 6 and 60 min of growth, respectively. (d) SEM picture from the final sample grown for $60 \mathrm{~min}$.

The growth mode changes from 2-dimensional layer-by-layer growth to 3D growth of nanowires. The evolution of RHEED diffraction images in such a case is shown in Fig. 12.

The Mn flux supplied during the MBE growth of the sample presented in Fig. 12 corresponds to the Mn content of 6 at.\% in the uniform GaMnAs layer. In such a case the density of nanowires is very high, probably due to the catalyzing action of MnAs clusters generated during the whole growth process both at the surface between nanowires and on their sides. This latter process leads to formation of branches. Lowering the Mn flux during the NW growth reduces the density of NWs, as shown in the SEM pictures in Fig. 13.

Figure 14 shows SEM pictures of individual nanowires removed from the sample grown with $1 \% \mathrm{Mn}$, and placed on a $\mathrm{Si}$ wafer.

There are still open questions concerning the properties of nanowires obtained in the GaMnAs MBE growth process at the MnAs segregation conditions. 


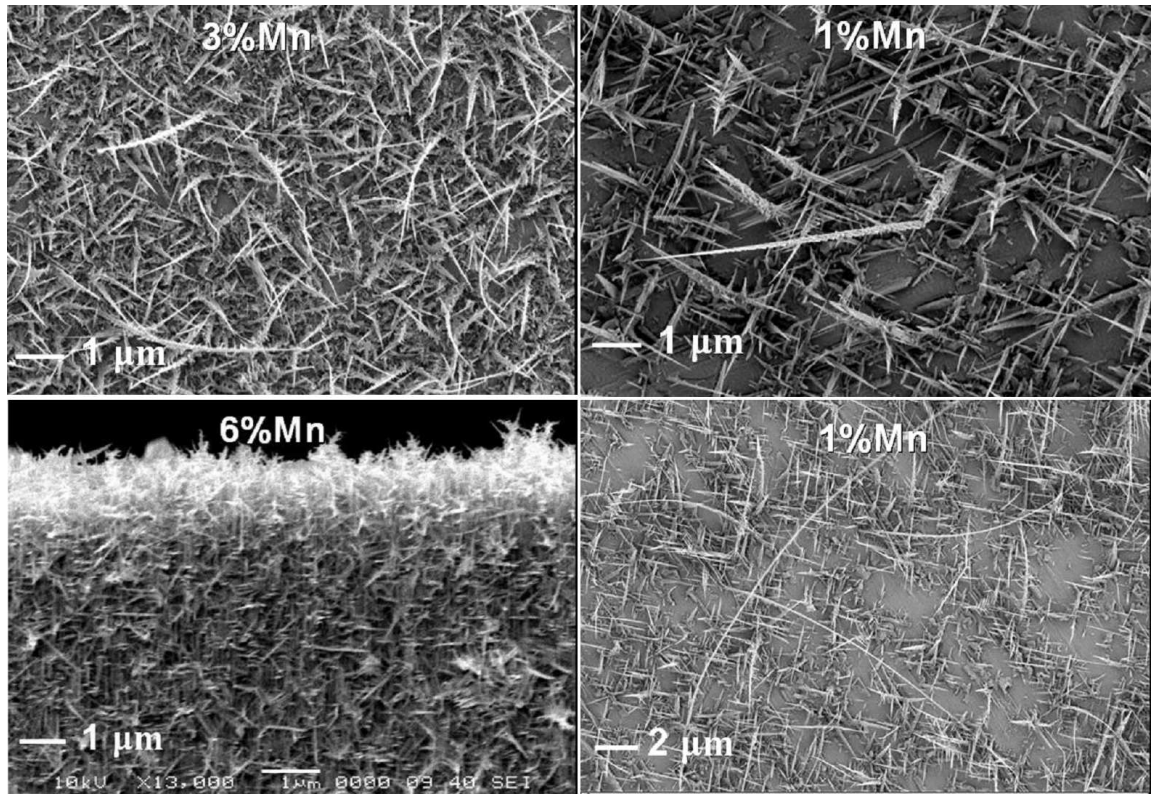

Fig. 13. SEM pictures of GaMnAs nanowires grown on $\operatorname{GaAs}(100)$ substrate at different Mn fluxes.
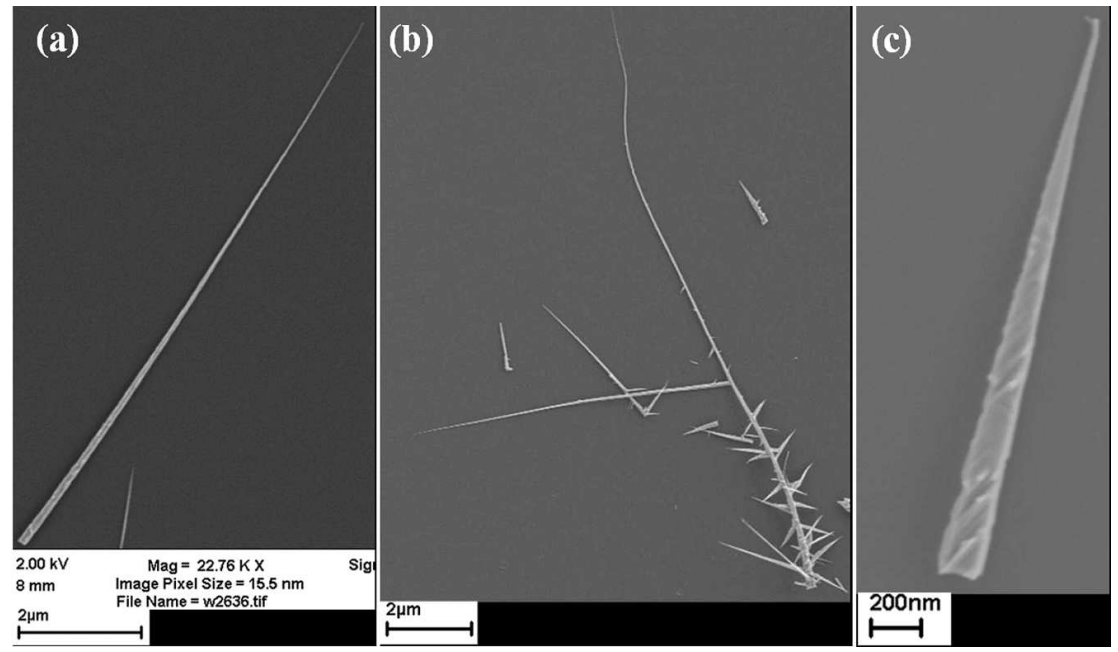

Fig. 14. Three types of nanowires separated from the sample grown with $1 \% \mathrm{Mn}$ : (a) long nanowire without branches, (b) long nanowire with branches, (c) short, tapered nanowire.

The as-grown samples with high density of nanowires were found to exhibit ferromagnetic properties [14], but the magnetism of individual nanowires still remains to be investigated. 


\section{Conclusions}

Post-growth annealing of GaMnAs samples with the presence of an As passivating layer has been used for removing of Mn interstitials. Annealing can be done in the MBE system directly after the MBE growth. It was shown that this post-growth treatment effectively increases ferromagnetic to paramagnetic phase transition temperature in GaMnAs. The efficiency of the procedure is higher for GaMnAs layers grown on InGaAs buffers. As a side effect of the annealing a MnAs-rich surface is formed, consisting of either very thin continuous layer of zinc-blende MnAs, or MnAs dots. In the first case it is possible to grow heterostructures containing GaMnAs and thin cubic MnAs. The latter case can be used to develop GaMnAs nanowires, where MnAs islands catalyze the nanowire growth. The MnAs nano-islands segregated at GaMnAs surface during the MBE growth above phase segregation threshold exhibit the same catalyzing properties.

\section{Acknowledgments}

The author would like to thank Dr. J.Z. Domagała from the Institute of Physics, Polish Academy of Sciences, Warsaw for the extensive collaboration in X-ray diffraction characterization of GaMnAs, E. Łusakowska for AFM measurements, Dr. H. Shtrikmann from Weizmann Institute, Rehovot for SEM measurements of nanowires, and Prof. J. Kanski from CTH, Göteborg for careful reading of the manuscript. The GaMnAs project at MAX-Lab, Lund is partially financed by the Swedish Research Council (VR). The financial support from EADS (France) research project coordinated by Prof. S. Charar and Dr. F. Terki, from GES Université Montpellier II; and acces to the Braun Center for Submicron Research at the Weizmann Institute, Rehovot financed through the EC project RITA-CT2003-506095 WISSMC is greatly acknowledged.

\section{References}

[1] H. Ohno, A. Shen, F. Matsukura, A. Oiwa, A. Endo, S. Katsumoto, Y. Iye, Appl. Phys. Lett. 69, 363 (1996).

[2] S.J. Potashnik, K.C. Ku, S. J. Potashnik, K.C. Ku, S.H. Chun, J.J. Berry, N. Samarth, P. Schiffer, Appl. Phys. Lett. 79, 1495 (2000).

[3] K.M. Yu, W. Walukiewicz, T. Wojtowicz, I. Kuryliszyn, X. Liu, Y. Sasaki, J.K. Furdyna, Phys. Rev. B 65, 20130 (2002).

[4] V. Avrutin, D. Humienik, S. Frank, A. Koeder, W. Schoch, W. Limmer, R. Sauer, A. Waag, J. Appl. Phys. 98, 023909 (2005).

[5] K.W. Edmonds, K.Y. Wang, R.P. Campion, A.C. Neumann, N.R.S. Farley, B.L. Gallagher, C.T. Foxon, Appl. Phys. Lett. 81, 4991 (2002).

[6] K.W. Edmonds, P. Boguslawski, K.Y. Wang, R.P. Campion, N. Novikov, N.R.S. Farley, B.L. Gallagher, C.T. Foxon, M. Sawicki, T. Dietl, M. Buongiorno Nardelli, J. Bernholc, Phys. Rev. Lett. 92, 037201 (2004).

[7] K. Olejnik, M.H.S. Owen, V. Novak, J. Masek, A.C. Irvine, J. Wunderlich, T. Jungwirth, arxiv.cond-mat.0802.2080 (2008). 
[8] S. Ohya, K. Ohno, M. Tanaka, Appl. Phys. Lett. 90, 112503 (2007).

[9] D. Chiba, Y. Nishitani, F. Matsukura, H. Ohno, Appl. Phys. Lett. 90, 122503 (2007).

[10] S. Mack, R.C. Myers, J.T. Heron, A.C. Gossard, D.D. Awschalom, Appl. Phys. Lett. 92, 192502 (2008).

[11] C. Rüster, T. Borzenko, C. Gould, G. Schmidt, L.W. Molenkamp, X. Liu, T.J. Wojtowicz, J.K. Furdyna, Z.G. Yu, M.E. Flatté, Phys. Rev. Lett. 91, 216602 (2003).

[12] D. Neumaier, K. Wagner, S. Geißler, U. Wurstbauer, J. Sadowski, W. Wegscheider, D. Weiss, Phys. Rev. Lett. 99, 116803 (2007).

[13] M. Ciorga, M. Schlapps, A. Einwanger, S. Geißler, J. Sadowski, W. Wegscheider, D. Weiss, New J. Phys. 9, 351 (2007)

[14] J. Sadowski, P. Dłużewski, S. Kret, E. Janik, E. Łusakowska, J. Kanski, A. Presz, F. Terki, S. Charar, D. Tang, Nano Lett. 7, 2724 (2007).

[15] P. Dłużewski, S. Kret, J. Sadowski, to be published.

[16] T.E.M. Staab, R.M. Nieminen, J. Gebauer, R. Krause-Rehberg, M. Luysberg, M. Haugk, Th. Frauenheim, Phys. Rev. Lett. 87, 045504 (2001).

[17] R.P. Campion, K.W. Edmonds, L.X. Zhao, K.Y. Wang, C.T. Foxon, B.L. Gallagher, C.R. Staddon, J. Cryst. Growth 247, 42 (2003).

[18] K.M. Yu, W. Walukiewicz, T. Wojtowicz, I. Kuryliszyn, X. Liu, Y. Sasaki, J.K. Furdyna, Phys. Rev. B 65, 201303(R) (2002).

[19] M. Adell, L. Ilver, J. Kanski, V. Stanciu, P. Svedlindh, J. Sadowski, J.Z. Domagala, F. Terki, C. Hernandez, S. Charar, Appl. Phys. Lett. 86, 112501 (2005).

[20] M. Adell, J. Adell, J. Kanski, J. Sadowski, J.Z. Domagała, Phys. Rev. B 75, 054415 (2007)

[21] J. Masek, J. Kudrnovsky, F. Maca, Phys. Rev. B 67, 153203 (2003).

[22] J. Sadowski, M. Adell, J. Kanski, L. Ilver, E. Janik, E. Lusakowska, J.Z. Domagała, S. Kret, P. Dłużewski, R. Brucas, M. Hanson, Appl. Phys. Lett. 87, $263114(2005)$. 